\title{
von Hippel-Lindau mutation in mice recapitulates Chuvash polycythemia via hypoxia-inducible factor-2 $\alpha$ signaling and splenic erythropoiesis
}

\author{
Michele M. Hickey,1,2,3 Jennifer C. Lam,1,3 Natalie A. Bezman,1,2,3
}

W. Kimryn Rathmell, ${ }^{4}$ and M. Celeste Simon ${ }^{1,2,3}$

\begin{abstract}
${ }^{1}$ Abramson Family Cancer Research Institute, ${ }^{2}$ Cell and Molecular Biology Graduate Group, and ${ }^{3}$ Howard Hughes Medical Institute, University of Pennsylvania School of Medicine, Philadelphia, Pennsylvania, USA. ${ }^{4}$ Lineberger Comprehensive Cancer Center, University of North Carolina, Chapel Hill, North Carolina, USA.
\end{abstract}

\begin{abstract}
The R200W mutation in the von Hippel-Lindau (VHL) tumor suppressor protein (PVHL) is unique in that it is not associated with tumor development, but rather with Chuvash polycythemia, a heritable disease characterized by elevated hematocrit and increased serum levels of erythropoietin and VEGF. Previous studies have implicated hypoxia-inducible factor-1 $\alpha$ (HIF-1 $\alpha)$ signaling in this disorder, although the effects of this mutation on $\mathrm{PVHL}$ function are not fully understood. In order to explore the mechanisms underlying the development of this polycythemia, we generated mice homozygous for the R200W mutation $\left(V h l^{R / R}\right)$. $V h l^{R / R}$ mice developed polycythemia highly similar to the human disease. The activity of HIF proteins, specifically the HIF-2 $\alpha$ isoform, was upregulated in ES cells and tissues from $V h l^{R / R}$ mice. Furthermore, we observed a striking phenotype in $V b l^{R / R}$ spleens, with greater numbers of erythroid progenitors and megakaryocytes and increased erythroid differentiation of $V b l^{R / R}$ splenic cells in vitro. These findings suggest that enhanced expression of key HIF-2 $\alpha$ genes promotes splenic erythropoiesis, resulting in the development of polycythemia in $V b l^{R / R}$ mice. This mouse model is a faithful recapitulation of this $V H L$-associated syndrome and represents a useful tool for studying polycythemias and investigating potential therapeutics.
\end{abstract}

\section{Introduction}

The von Hippel-Lindau (VHL) tumor suppressor protein (pVHL) is best-characterized as the substrate recognition component of an E3 ubiquitin ligase complex that targets hypoxia-inducible factors (HIFs) for degradation in an $\mathrm{O}_{2}$-dependent manner (1-5). HIF proteins mediate cellular adaptation to low $\mathrm{O}_{2}$, or hypoxia, by activating the expression of genes essential for processes including increased glucose uptake, enhanced red blood cell production, and angiogenesis. HIFs are heterodimers consisting of an $\alpha$ and a $\beta$ subunit (also known as aryl hydrocarbon receptor nuclear translocator) that bind to conserved sequences within the promoters or enhancers of target genes (6). The 2 primary HIF- $\alpha$ isoforms, HIF- $1 \alpha$ and HIF- $2 \alpha$, share many target genes but selectively activate others. For example, HIF- $1 \alpha$ regulates glycolytic enzymes such as phosphoglycerate kinase (PGK), whereas HIF- $2 \alpha$ regulates genes encoding Oct- 4 and erythropoietin (EPO) (7-10). The expression of other targets, including VEGF, glucose transporter-1 (GLUT-1), and plasminogen activator inhibitor-1 (PAI-1), is preferentially activated by HIF- $2 \alpha$ in certain cell types $(7,11,12)$.

Nonstandard abbreviations used: BFU-E, burst-forming unit(s)-erythroid; CFU-E, CFU-erythroid; CFU-G, CFU-granulocyte; CFU-GEMM, CFU-granulocyte-erythroidmacrophage-megakaryocyte; CFU-GM, CFU-granulocyte-macrophage; CFU-M, CFUmacrophage; EB, embryoid body; EPO, erythropoietin; Glut-1, glucose transporter-1; HIF, hypoxia-inducible factor; neo ${ }^{\mathrm{R}}$, neomycin resistance (cassette); PAI-1, plasminogen activator inhibitor-1; PGK, phosphoglycerate kinase; PHZ, phenylhydrazine; PVHL, VHL tumor suppressor protein; VHL, von Hippel-Lindau.

Conflict of interest: The authors have declared that no conflict of interest exists. Citation for this article: J. Clin. Invest. 117:3879-3889 (2007). doi:10.1172/JCI32614
Germline mutation of $1 \mathrm{VHL}$ allele is associated with a hereditary cancer syndrome known as VHL disease, in which patients are predisposed to highly vascular tumors that develop upon inactivation of the remaining $V H L$ allele in somatic tissue (13). VHL is also mutated in a majority of sporadic hemangioblastomas and renal clear cell carcinomas (14). Loss of pVHL function mimics a hypoxic state in which HIF is constitutively stabilized and inappropriately activates genes that mediate increased cell proliferation and angiogenesis (4). In addition, pVHL has been proposed to mediate HIF-independent functions that may be important for tumor suppression, including regulation of extracellular matrix assembly via direct binding of PVHL to fibronectin and suppression of JunB activity to promote apoptosis (15-18).

There is a strong genotype-phenotype correlation in VHL disease, such that the location of the mutation site in VHL determines the type of tumors that arise $(13,14)$. Most $V H L$ point mutations result in dysregulation of the HIF pathway, either by disrupting the formation of the E3 ubiquitin ligase complex or by impairing the ability of pVHL to bind HIFs (14). In contrast to these mutations however, germline homozygosity for the $598 \mathrm{C} \rightarrow T$ mutation in exon 3 of $V H L$ does not predispose to tumor formation, but instead is linked to an autosomal-recessive disease known as Chuvash polycythemia (19-21). Interestingly, polycythemia is not frequently observed in VHL disease, although it can occur secondary to EPO production by tumors and in mice with hepatic $V h l$ deletion or constitutive expression of HIF-2 $\alpha(12,13,22,23)$. This polycythemia is endemic to the Chuvash region of Russia and the Italian island of Ischia, but has also been identified in diverse ethnic backgrounds (24-28). The disease is characterized by elevated hemo- 

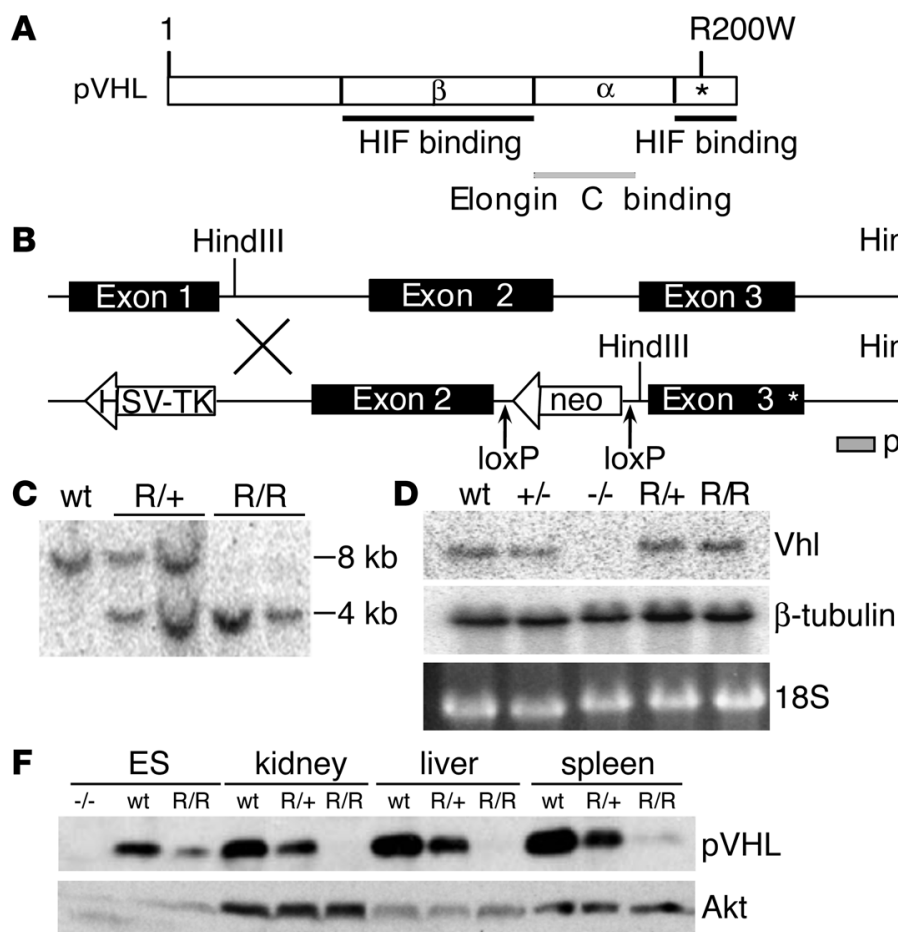

HindIII wt $V h l$ locus targeting construct

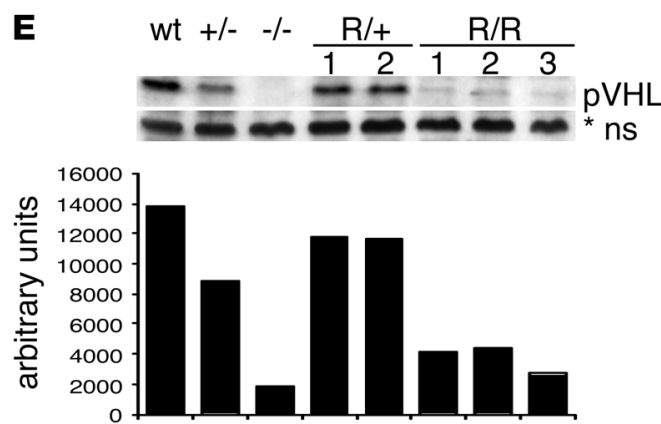

\section{Figure 1}

Generation of $V h / R / R$ ES cells. (A) Schematic representation of human pVHL showing the location of the R200W mutation (asterisk). (B) Targeting construct used to introduce the R200W mutation in murine ES cells by homologous recombination. (C) Southern blot genotyping of ES cells using the probe indicated in $\mathbf{B}$, showing heterozygous mutant $V h / R /+(R /+)$ and homozygous mutant $V h / R / R(R / R)$ clones. (D) Northern blot analysis using the probe indicated in B, confirming that the $V h /$ transcript in $V h / R / R$ cells was the same size as that in WT cells. (E) Western blot demonstrating that expression of $\mathrm{pVHL}$ in Vh/R/R cells was decreased compared with WT cells. As quantitated below, mutant $\mathrm{pVHL}$ levels were $30 \%-40 \%$ those of WT. *ns, nonspecific band for loading control. (F) Expression of mutant pVHL was also significantly decreased in the kidney, liver, and spleen of $V h /^{R / R}$ mice compared with WT and $V h /^{R /+}$ littermates.

globin and hematocrit, hypersensitivity of erythroid progenitors to EPO, and increased serum levels of EPO, VEGF, endothelin-1, and PAI-1 $(19,21,25,26,28,29)$. In addition, this unique type of polycythemia is associated with an increased risk of thrombotic complications, cerebral vascular events, pulmonary hypertension, and premature mortality $(21,25,30,31)$.

The 598C $\rightarrow$ T mutation results in a substitution of tryptophan for arginine at codon 200 (R200W in humans) at the extreme C terminus of pVHL (Figure 1A) (19, 20). This region of the protein is composed of an $\alpha$ helix that stabilizes the interaction between pVHL and its substrate and is required for complete HIF- $\alpha$ ubiquitination (32). Studies using B lymphocytes isolated from patients have demonstrated that this mutation diminishes, but does not completely abolish, the ability of pVHL to efficiently bind to and regulate HIF- $\alpha$ activity. Therefore, HIF- $\alpha$ proteins are moderately stabilized under normoxic conditions and upregulate the expression of genes such as EPO that likely contribute to the disease phenotype (19). However, previous studies have been limited to the use of transformed cells from humans, and multiple questions remain, including the extent to which dysregulation of HIF contributes to the polycythemic phenotype and which tissues are affected by this mutation. Furthermore, it is not clear how this mutation results in polycythemia without inducing tumor formation.

In order to elucidate the mechanisms underlying this disease and better understand how the Chuvash mutation affects pVHL function, we generated mice homozygous for the R200W muta- tion $\left(V h l^{R / R}\right)$. The phenotype of $V b l^{R / R}$ mice faithfully recapitulates many aspects of human Chuvash polycythemia, including elevated hematocrit and serum VEGF and EPO levels, and provides the most accurate murine model of a VHL-associated syndrome to date. Analysis of $V h l^{R / R}$ mice supported the importance of increased HIF signaling, in particular HIF-2 $\alpha$, and upregulation of key genes, such as those encoding VEGF, EPO, GLUT-1, and PAI-1, in the development of polycythemia. Moreover, erythroid differentiation was enhanced specifically in $V h l^{R / R}$ spleens, offering important insights into the pathology of Chuvash polycythemia and potential therapeutic implications.

\section{Results}

Generation of $V h l^{R / R}$ ES cells. To better study the effect of the Chuvash mutation on PVHL function and polycythemia development, we first generated murine ES cells expressing the R200W mutation (R166W in the mouse). This mutation is located at the extreme $\mathrm{C}$ terminus of $\mathrm{PVHL}$, in a region important for HIF- $\alpha$ binding (Figure $1 \mathrm{~A}$ ) (32). Figure $1 \mathrm{~B}$ shows the targeting construct used to introduce this point mutation in exon 3 of the endogenous $V b l$ locus by homologous recombination. Heterozygous $\left(V h l^{R /+}\right)$ clones were identified by both PCR (data not shown) and Southern blot analysis with the indicated probe (Figure 1, B and C). Selection of ES cells with increasing concentrations of G418 produced homozygous R200W clones $\left(V h l^{R / R}\right.$; Figure 1C) (33). The Vhl locus was sequenced to ensure that no additional mutations were introduced, and Northern blot- 

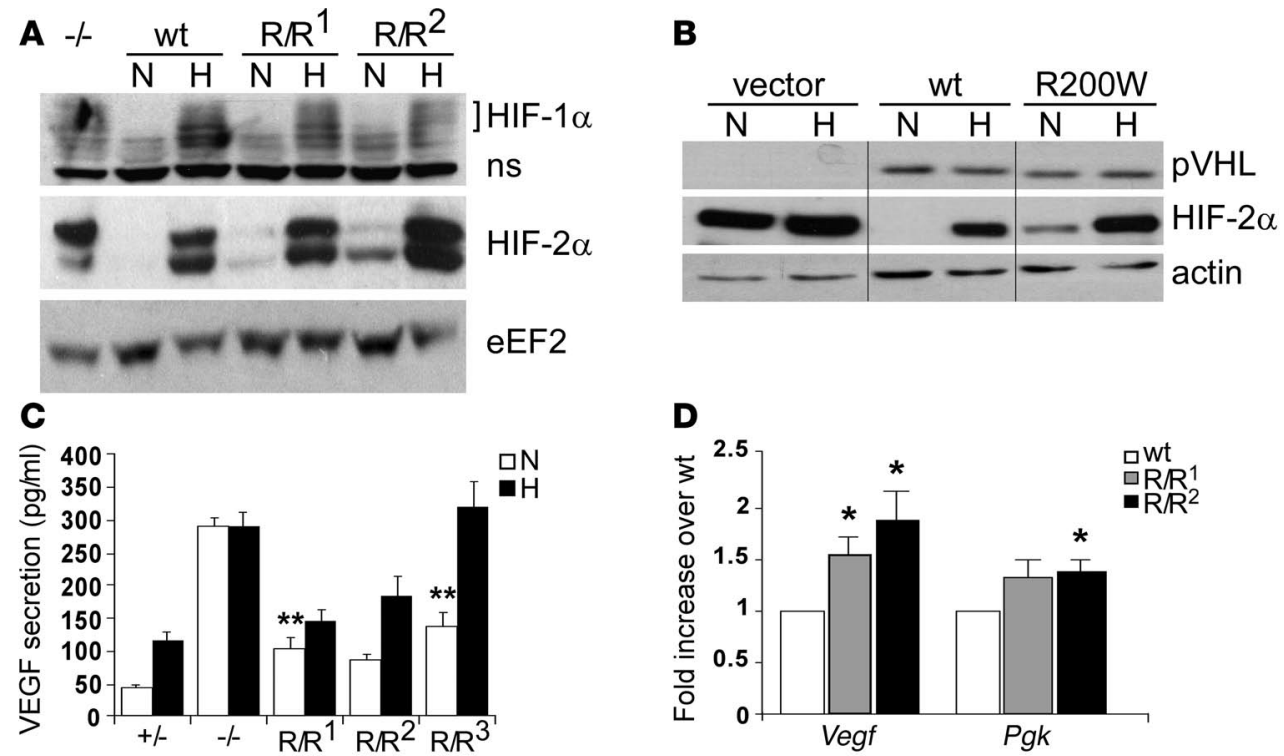

Figure 2

HIF activity is increased in Vh/R/R ES cells. (A) Western blot analysis for HIF-1 $\alpha$ and HIF-2 $\alpha$ in WT or 2 $V h / R / R$ clones $\left(R / R^{1}\right.$ and $\left.R / R^{2}\right)$ grown under either normoxia $(\mathrm{N})$ or $1.5 \%$ hypoxia $(\mathrm{H})$. HIF-1 $\alpha$ protein was not detectably stabilized under normoxia; however, normoxic HIF-2 $\alpha$ protein levels were greater in Vh/R/R cells than in WT cells. (B) Stable clones of 786-O human renal carcinoma cells expressing murine WT Vhl or VhlR20oW (R200W) were generated and screened by Western blot for equivalent expression of WT and R200W pVHL. These samples were run on the same gel but were noncontiguous, as indicated by black lines. Normoxic levels of HIF-2 $\alpha$ were increased in R200W, but not WT, clones. (C) Secretion of VEGF by ES cells as measured by ELISA. Hypoxic induction of VEGF protein was maintained in $V h /^{R / R}$ cells, similar to control heterozygous (+/-) cells. However, normoxic VEGF protein levels were increased 2- to 3-fold in 3 independent $V h l^{R / R}$ clones compared with control heterozygous cells. ${ }^{*} P<0.0006$. (D) WT or $V h l^{R / R}$ ES cells were grown in methylcellulose and allowed to differentiate into EBs for 9 days. The expression of Vegf and Pgk was analyzed by TaqMan real-time PCR. VEGF mRNA levels were increased 1.5- to 2-fold in $V h / R / R$ EBs over WT, while PGK was upregulated 1.3-fold. ${ }^{*} P<0.03$.

ting confirmed the integrity of the targeted mRNA (Figure 1D). The neomycin resistance $\left(n e o^{R}\right)$ cassette was removed in vitro by electroporation of a Cre recombinase-expressing plasmid followed by PCR and Southern blot screening.

Surprisingly, mutant R200W pVHL levels were decreased to approximately $30 \%$ those of WT in multiple independent ES clones (Figure 1E), and also in tissues from $V h l^{R / R}$ mice (Figure $1 \mathrm{~F}$ ). The half-life of mutant $\mathrm{PVHL}$ was also reduced compared with the WT protein (Supplemental Figure 1A; supplemental material available online with this article; doi:10.1172/JCI32614DS1). To examine possible reasons for this reduction, we performed a number of control assays. To confirm that the perceived decrease did not result from loss of an antibody epitope, we compared the ability of 2 different antibodies to detect in vitro transcribed and translated pVHL. Whereas an antibody generated against the $\mathrm{C}$ terminus of pVHL was unable to detect R200W pVHL as efficiently as WT, an antibody to full-length $\mathrm{PVHL}$ recognized both proteins equivalently (Supplemental Figure 1B). Therefore, we used this full-length antibody for all subsequent experiments. In addition, we compared levels of in vitro transcribed and translated WT and R200W pVHL labeled with ${ }^{35} \mathrm{~S}$ and found no difference, arguing against a deficit in translation (Supplemental Figure 1C). Treatment of WT or $V b l^{R / R}$ ES cells with various proteasome inhibitors (ALLN, lactacystin, and MG132), however, resulted in somewhat increased mutant pVHL levels, suggesting that the R200W mutation renders pVHL less stable and more susceptible to degradation compared with WT (Supplemental Figure 1D).

Analysis of HIF regulation in $\mathrm{Vh} l^{R / R}$ ES cells. To determine the effect of the R200W mutation on the HIF pathway, we examined HIF-1 $\alpha$ and HIF- $2 \alpha$ protein levels. As shown in Figure 2A, hypoxic induction of both HIF- $1 \alpha$ and $\mathrm{HIF}-2 \alpha$ in $V h l^{R / R}$ ES cells was similar to that seen in WT cells. However, although normoxic HIF-1 $\alpha$ protein was not detectably increased in $V h l^{R / R}$ ES cells, we noted a reproducible increase in the level of normoxic HIF-2 $\alpha$ (Figure 2A). In order to exclude the possibility that increased HIF- $2 \alpha$ was simply the result of reduced $\mathrm{PVHL}$ levels, we generated stable clones of 786-O human renal carcinoma cells (which lack functional pVHL) stably expressing either WT or R200W pVHL at similar levels (Figure 2B). Importantly, normoxic HIF-2 $\alpha$ protein was exclusively detected in cells expressing R200W pVHL, demonstrating that aberrant HIF regulation results from the effects of this point mutation on pVHL function (Figure 2B).

HIF target gene expression was also increased in $V b l^{R / R}$ cells under normoxia, further indicating that the R200W mutation results in enhanced HIF activity. Normoxic secretion of VEGF protein by $V h l^{R / R}$ ES cells was approximately 2- to 3-fold greater than that of control heterozygous cells (Figure 2C). The effect of the R200W point mutation on HIF and VEGF regulation was less severe than loss of $\operatorname{Vbl}(4,13)$, and $V h l^{R / R}$ ES cells maintained the ability to hypoxically induce VEGF expression similar to that of controls (Figure 2C). To further investigate HIF target gene expression, WT or $V b l^{R / R} \mathrm{ES}$ cells were cultured in methylcellulose and allowed to differentiate into embryoid bodies (EBs). TaqMan real-time PCR analysis performed on EB RNA demonstrated that Vegf and Pgk expression were approximately 1.5 - to 2 -fold and 1.3 -fold greater, respectively, in $V h l^{R / R} \mathrm{EBs}$ (Figure 2D). These findings provide evidence that in the presence of mutant pVHL, stabilized HIF- $2 \alpha$ is competent to moderately activate transcription of target genes in vitro.

\section{Table 1}

Genotypic frequencies of mice and embryos

\begin{tabular}{lcccc} 
& WT & $\boldsymbol{V h}^{\boldsymbol{R} / \boldsymbol{+}}$ & $\boldsymbol{V h}^{\boldsymbol{R} / \boldsymbol{R}}$ & Total \\
Live-born pups & 62 & 109 & 38 & 209 \\
Expected & 52.25 & 104.5 & 52.25 & \\
\hline
\end{tabular}

The frequency of live-born $V h /^{R / R}$ pups compared with the expected frequency was not significant $(P>0.1)$. 
A

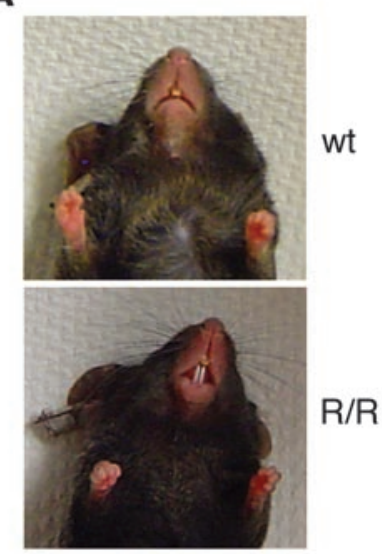

C

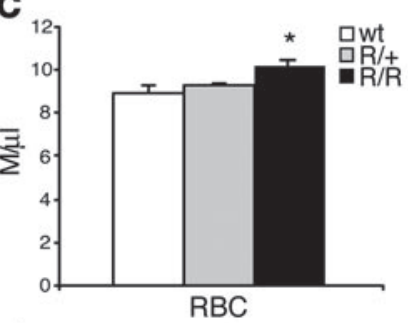

E

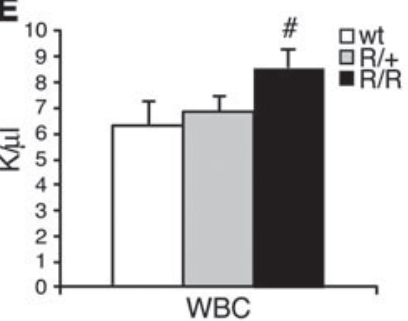

B

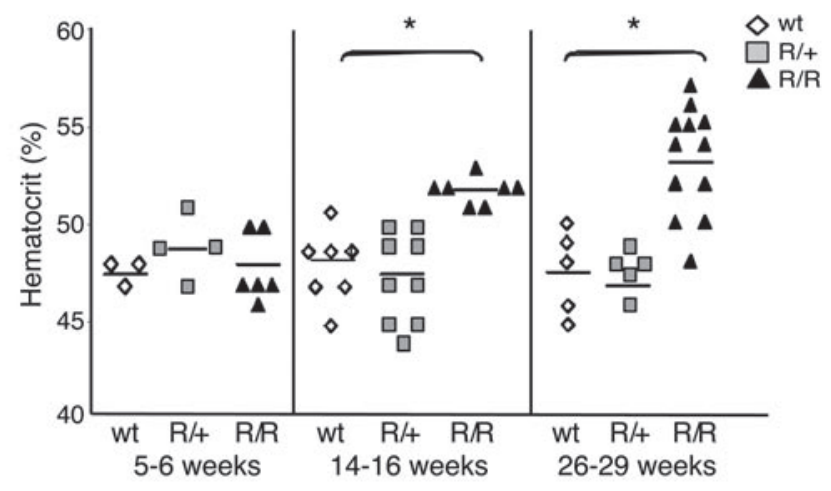

D

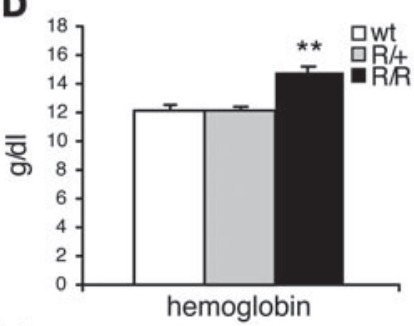

$\mathbf{F}$

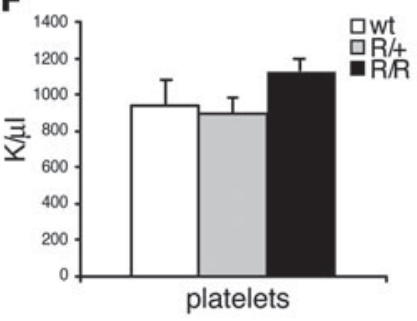

Figure 3

$V h R^{R / R}$ mice develop polycythemia. (A) The snout and paws of $V h / R / R$ mice appeared redder in color than WT littermates. (B) There was no difference in the hematocrit levels based on genotype at 5-6 weeks; however, the hematocrit of $V h I^{R / R}$ mice became significantly greater than WT and $V h /^{R /+}$ mice with increasing age $(9 \%$ higher at 14-16 weeks and $11.8 \%$ higher at 26-29 weeks). ${ }^{*} P<0.0005$. (C and D) Total red blood cell number (C) and hemoglobin concentration (D) were $14 \%$ and $22 \%$ greater, respectively, in $V h / R / R$ mice than in WT mice. ${ }^{*} P<0.004 ;{ }^{* *} P<0.0007$. (E) Total white blood cell number was also increased by $36 \%$ in $V h R^{R / R}$ mice. ${ }^{\#} P<0.059$ (trending toward significance). (F) The number of platelets was not significantly increased in $V h /^{R / R}$ mice. Results in $\mathbf{C}-\mathbf{F}$ represent pooled data from mice aged 9-15 months.
$V h l^{R / R}$ mice develop polycythemia. $V b l^{R /+}$ ES cells were injected into blastocysts to generate founder mice, which were genotyped by the same PCR and Southern blot methods used for ES cells. These mice were then crossed to E2A Cre transgenics to remove the neo $o^{R}$ cassette (34). Interbreeding of heterozygous mice yielded viable homozygous $V h l^{R / R}$ pups, although at a slightly lower frequency than expected (Table 1), suggesting that this $V b l$ point mutation has deleterious, but variable, effects during mouse embryogenesis. However, we focused our attention on phenotypic characterization of live-born $V b l^{R / R}$ mice.

$V h l^{R / R}$ mice developed redness in their snouts and paws, as shown in Figure 3A. This was similar to other mice with polycythemia $(12,23)$ and suggested that $V h l^{R / R}$ mice may be polycythemic. To confirm this, we measured hematocrits of age-matched WT, $V h l^{R /+}$, and $V h l^{R / R}$ mice at various ages. At 5-6 weeks there was no difference in hematocrit levels based on genotype, but a significant increase in hematocrits of $V h l^{R / R}$ mice became apparent as the mice aged (Figure 3B). $V h l^{R / R}$ mice first developed elevated hematocrits by approximately $10-14$ weeks after birth, and levels continued to increase to nearly $55 \%$ on average by 26 weeks (Figure 3B). In contrast, hematocrits of both WT and $V h l^{R /+}$ mice remained relatively constant over time (averaging 47\%; Figure 3B). Therefore, $V b l^{R / R}$ mice are in fact polycythemic, with a moderate increase in hematocrit similar to that seen in humans with this mutation $(21,25)$. As is also the case for human R200W homozygotes (21), we observed no increased predisposition to tumor formation in $V h l^{R / R}$ mice.
In addition to elevated hematocrits, red blood cell numbers and hemoglobin levels were significantly increased in $V h l^{R / R}$ animals relative to WT and $V h l^{R /+}$ littermates (Figure 3, C and D). Reticulocyte counts, however, were not changed in $V h l^{R / R}$ mice (data not shown). The R200W mutation may also have a more general effect on hematopoietic differentiation, at least in mice: circulating white blood cell numbers were slightly higher $(P<0.059$; trending toward significance) in $V h l^{R / R}$ mice (Figure $3 \mathrm{E}$ ), although platelet counts appeared normal (Figure $3 \mathrm{~F}$ ).

$H I F$ target genes are upregulated in $V h l^{R / R}$ mice. To further explore the extent to which the polycythemia we observed resembled Chuvash polycythemia, serum VEGF and EPO levels were assayed by ELISA. The concentration of both proteins was greater - by approximately 1.3-fold $(P<0.002)$ and 1.8 -fold $(P<0.08)$, respectively - in $V h l^{R / R}$ serum compared with WT controls (Figure 4, A and B). In addition, $V h l^{R / R}$ serum levels of both VEGF and EPO increased further with age (Supplemental Figure 2, A and B), consistent with the elevated hematocrits observed in older mice (Figure 3B).

The results of serum ELISAs and increased HIF- $2 \alpha$ protein levels observed in ES cells implicated HIF signaling as a major mechanism underlying the development of polycythemia. To investigate this possibility further, we measured mRNA levels of HIF target genes in representative tissues isolated from $V h l^{R / R}$ mice and corresponding littermate controls (Figure 4, C-F). Epo mRNA levels in $V h l^{R / R}$ kidneys were slightly increased (1.3-fold; Figure 4D), suggest- 

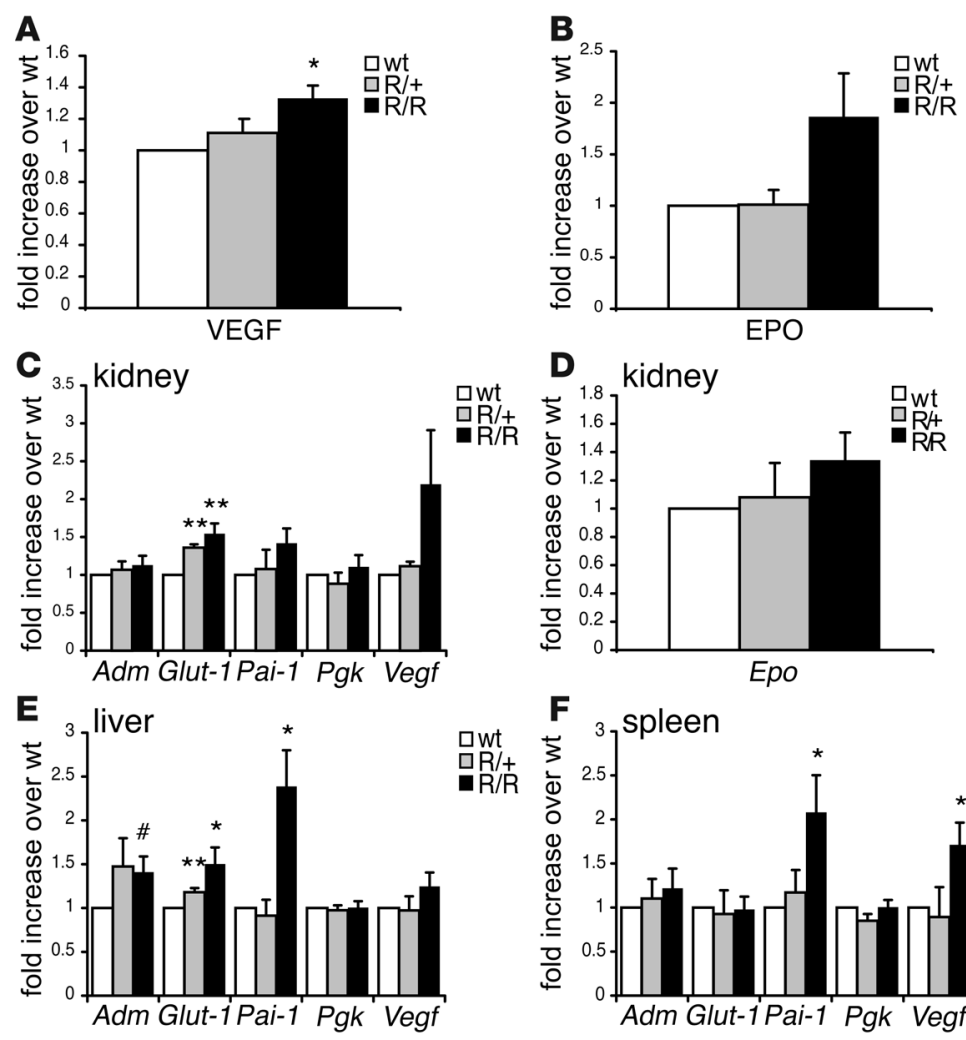
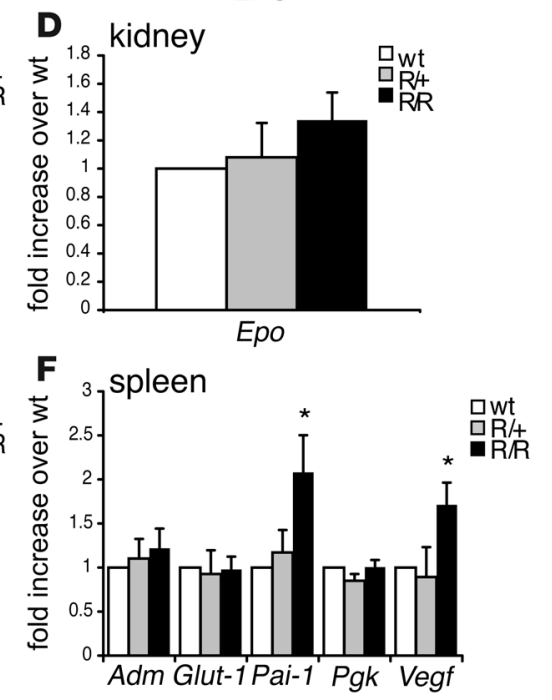

Figure 4

HIF target gene expression is increased in $V h l^{R / R}$ mice. (A and $\mathbf{B})$ Serum levels of VEGF and EPO were determined by ELISA; pooled results from mice of varying ages are shown. (A) VEGF levels were 1.3-fold greater in $V h l^{R / R}$ mice than in WT and $V h l^{R /+}$ mice. ${ }^{*} P<0.002$. (B) EPO levels were 1.8-fold greater in $V h l^{R / R}$ mice $(P<0.08)$. (C-F) TaqMan real-time PCR analysis of HIF gene expression in kidney (C and $\mathbf{D})$, liver (E), and spleen $(\mathbf{F})$. The expression of several genes, most notably HIF-2 $\alpha$-preferential targets such as Glut-1, $P A l-1$, and VEGF, was increased in tissues isolated from $V h l^{R / R}$ mice compared with those of WT mice. ${ }^{\star} P<0.05 ;{ }^{\star} P<0.007 ;{ }^{\#} P<0.059$ (trending toward significance). Renal Epo mRNA expression was increased 1.3-fold $(P<0.09)$ in $V h l^{R / R}$ mice with increased serum EPO levels (D). ing that renal Epo production is the likely source of the increased serum EPO in these mice. Importantly, Epo expression was undetectable in the liver, brain, and spleen of both WT and $V h l^{R / R}$ mice (data not shown). Vegf expression was upregulated in each tissue tested, most notably in the spleen (1.7-fold; Figure 4, C, E, and F). Similarly, Glut-1 (Slc2a1) mRNA levels were elevated in $V b l^{R / R}$ kidneys and livers (Figure 4, C and E). In addition, the expression of other HIF target genes likely to contribute to the observed phenotype was enhanced in $V h l^{R / R}$ tissues. The expression of mRNA encoding PAI-1, which negatively regulates the fibrinolytic cascade, was most significantly increased among the genes tested, with 2.4-fold greater expression in $V h l^{R / R}$ livers and 2.1-fold greater expression in $V h l^{R / R}$ spleens (Figure 4, E and F). Endothelin-1 $(E d n 1)$ mRNA levels were also greater in $V b l^{R / R}$ kidneys (2.3-fold), as was transferrin (Trf) expression in $V h l^{R / R}$ livers (1.4-fold; data not shown), consistent with the phenotype of Chuvash polycythemia $(21,29)$. Strikingly, expression of the HIF-1 $\alpha$-specific target gene $P g k$ was similar in $V h l^{R / R}$ and WT mice, whereas genes preferentially activated by HIF- $2 \alpha$, such as those encoding VEGF, EPO, and PAI-1 $(7,11,12)$, were upregulated in $V h l^{R / R}$ mutant mice (Figure 4, $\mathrm{C}-\mathrm{F})$. This distinction further suggests that HIF- $2 \alpha$ regulation may be more sensitive to the R200W point mutation.

Bone marrow is not affected in $V h l^{R / R}$ mice. The R200W mutation is ubiquitously expressed in all cells, and it is not known which stages of hematopoiesis are dysregulated, or whether the bone marrow and spleen are differentially affected by this mutation. We performed flow cytometry to examine whether more erythroid progenitor cells were present in $V h l^{R / R}$ mice using 2 markers of the maturing erythroid lineage, Ter119 and CD71 (transferrin receptor). Ter119 is an erythroid-specific marker expressed at all stages, whereas CD71 expression is highest on early erythroid precursors and is subse- quently lost as these cells differentiate to form mature red cells. There was a 2-fold higher percentage of circulating CD71 ${ }^{+}$Ter $119^{+}$ immature erythroid cells in $V h l^{R / R}$ blood than in WT and $V h l^{R /+}$ blood, suggesting that enhanced erythroid differentiation underlies polycythemia development in these mice (Figure 5, A and B). To determine the origin of these additional erythroid precursors in $V h l^{R / R}$ animals, we first analyzed cells from the bone marrow. However, the percentage of CD71 ${ }^{+}$Ter $119^{+}$cells in $V b l^{R / R}$ bone marrow was not significantly different from littermate controls (Figure 5, C and D). Furthermore, the histology of mutant bone marrow closely resembled that of WT, with similar architecture, cellularity, and numbers of hematopoietic progenitors (Figure 5E).

The R200W mutation stimulates erythropoiesis in the spleen. Given the absence of an effect on bone marrow erythropoiesis, we analyzed the spleen, a frequent site of extramedullary hematopoiesis in adult mice. $V h l^{R / R}$ spleens appeared darker in color than those of WT and $V h l^{R /+}$ littermates (Figure 6A). Histological analysis of $V h l^{R / R}$ spleens revealed disruption of normal splenic architecture, with decreased size of white pulp germinal centers and concomitant increases in red pulp containing many clusters of erythroid precursors (Figure 6B, white arrows). Of note, a striking abundance of megakaryocytes was detected in $V h l^{R / R}$ spleens, with a greater than 2 -fold increase compared with controls (Figure 6C). The megakaryocytes in $V h l^{R / R}$ spleens were visible even at low power and often appeared in clusters (Figure 6B, black arrows).

In agreement with our histological observations, $V h l^{R / R}$ spleens exhibited a greater than 6-fold increase in the percentage of $\mathrm{CD} 71^{+}$Ter $119^{+}$erythroid precursors (Figure 6, D and E) and decreased percentages of CD $19^{+}$B cells (WT, 53\%, Vhl $l^{R / R}, 44 \%$; Figure 6F). The percentage of cells positive for CD41 (a marker of megakaryocytes and platelets) was also higher in mutant spleens 


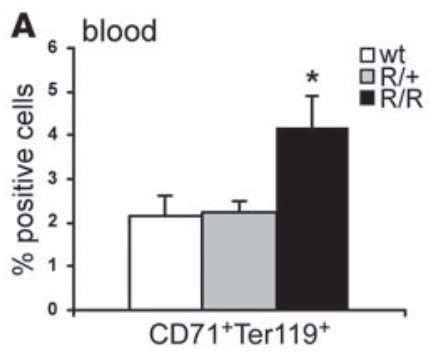

B
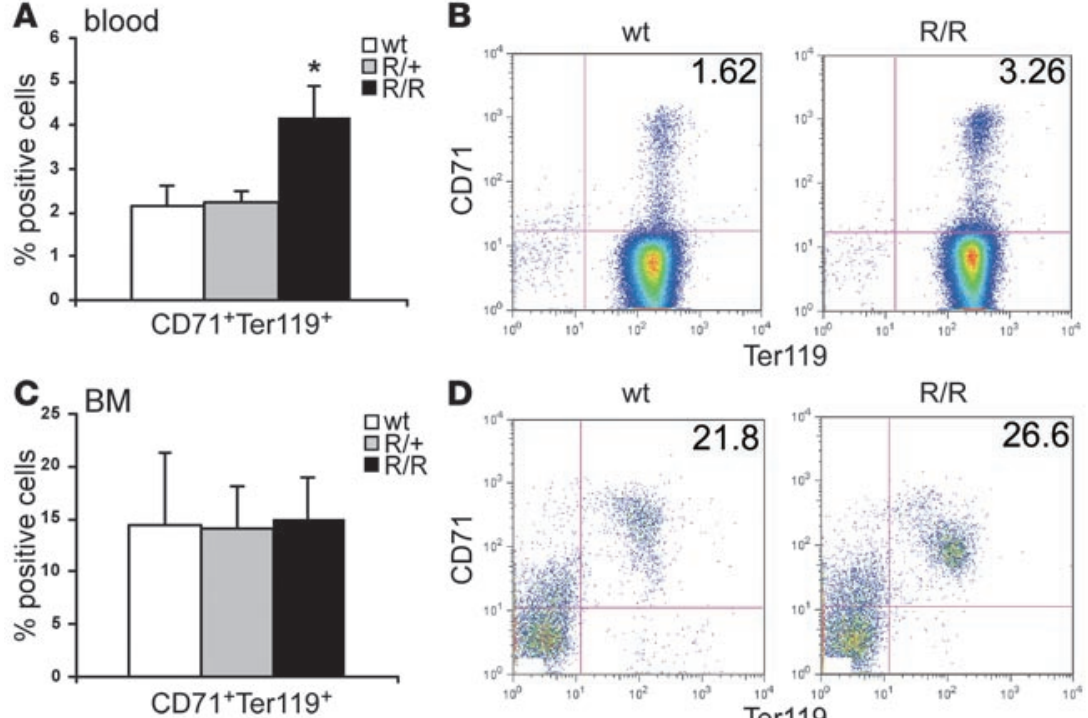

D

wt

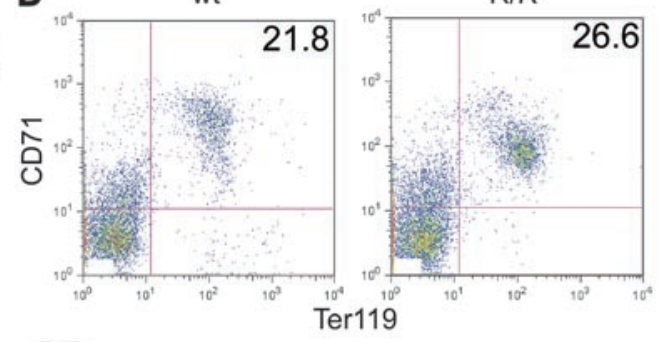

$\mathrm{R} / \mathrm{R}$

E

wt

\section{Figure 5}

Bone marrow is not the source of the increased erythroid progenitors in Vh/R/R mice. (A) Flow cytometry demonstrated a 2 -fold increase in the percentage of CD71+Ter119+ erythroid precursor cells in the blood of $V h l^{R / R}$ mice. ${ }^{*} P<0.03$. (B) Representative flow cytometry plots for WT and $V h /^{R / R}$ blood stained for CD71 and Ter119. (C) The percentage of CD71+Ter119+ cells was not significantly different in $V h / R / R$ bone marrow. (D) Representative flow cytometry plots for WT and Vh/R/R bone marrow stained for CD71 and Ter119. (E) Representative sections of WT and $V h / R / R$ bone marrow stained with $\mathrm{H} \& \mathrm{E}$ revealed no dramatic differences in bone marrow architecture. Original magnification, $\times 40$. Scale bars: $20 \mu \mathrm{m}$.

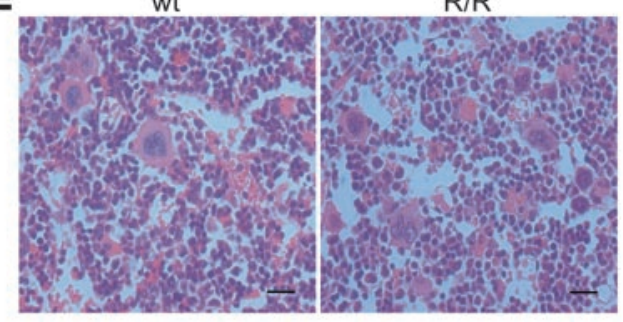

(2.5-fold), matching the observed increase in megakaryocytes (Figure $6 \mathrm{G})$. Taken together, these data indicate that increased erythroid precursor production in polycythemic $V h l^{R / R}$ mice occurs in the spleen and suggest that the R200W mutation may exert its effect on a hematopoietic precursor common to the erythroid and megakaryocytic lineages.

The above findings support the idea that in $V h l^{R / R}$ animals, the spleen serves as a reservoir of erythroid precursor cells in a basal, unstressed state. Therefore, we examined whether $V h l^{R / R}$ mice would respond more efficiently to erythropoietic stress. Mice were injected on 2 consecutive days with phenylhydrazine (PHZ) to induce hemolysis. As expected, prior to $\mathrm{PHZ}$ administration, $V h l^{R / R}$ mice had hematocrit levels $6 \%-27 \%$ greater than those of control animals $\left(V h l^{R / R}, 50 \%-56 \%\right.$; control, $44 \%-47 \%$; data not shown). PHZ treatment caused a comparable reduction in the hematocrits of all mice, decreasing hematocrit levels to $33 \%-37 \%$ at day 5 (Figure 7A). However, although all hematocrits were restored to pre-PHZ levels after approximately 1 week, $V h l^{R / R}$ mice were unable to downregulate the stress response upon reaching WT hematocrit values (Figure 7A). Instead, mutant mice inappropriately continued erythropoiesis, increasing their hematocrit to the polycythemic levels observed prior to $\mathrm{PHZ}$ (55\%-57\%; Figure 7A). In response to PHZ stress, all mice initiated erythropoiesis, resulting in an increase in splenic weight. Untreated $V h l^{R / R}$ spleens were already slightly larger than those of WT littermates (Figure 6A and Figure 7B). However, following $\mathrm{PHZ}$ treatment, a greater increase in the spleen/body weight ratios of $V h l^{R / R}$ mice was detected (untreated, $0.38 \%$; after PHZ, $0.64 \%$ ) compared with WT mice (untreated, $0.30 \%$; after PHZ, $0.43 \%)$, and the spleens of $V h l^{R / R}$ mice remained larger even 1 month after treatment (Figure 7B). Therefore, although the response to stress was not completely disrupted in mutant mice, PHZ further enhanced erythropoiesis in $V b l^{R / R}$ spleens.

Differentiation of multiple hematopoietic lineages is enhanced in $V h l^{R / R}$ mice. The above data suggested that the Vhl R200W mutation might promote increased differentiation of erythroid precursor cells, resulting in the development of polycythemia. To investigate this possibility, we evaluated hematopoietic differentiation using an in vitro colony-forming assay. Bone marrow or splenic cells from WT, $V h l^{R /+}$, or $V h l^{R / R}$ mice were plated in methylcellulose containing SCF, IL-3, IL-6, and EPO to support the formation of all hematopoietic lineages. The number of erythroid colonies formed by $V h l^{R / R}$ bone marrow was not different from that of WT or $V h l^{R /+}$ bone marrow (Figure 8A), in agreement with our flow cytometry and histological analyses (Figure 5, C-E). Similarly, there was no statistically significant change in the potential of $V b l^{R / R}$ bone marrow cells to generate colonies of other lineages, CFU-granulocyte (CFU-G), CFU-macrophage (CFU-M), CFU-granulocyte-macrophage (CFU-GM), or CFU-granulocyte-erythroid-macrophagemegakaryocyte (CFU-GEMM), although there was a trend toward increased CFU-G and CFU-M colonies (Figure 8, B and C).

In contrast, $V h l^{R / R}$ splenic cells exhibited an enhanced potential to form cells of various hematopoietic lineages, most notably erythroid colonies. We detected a 3.5-fold increase in the number of erythroid colonies formed by $V h l^{R / R}$ spleens compared with WT, as well as significant increases in the number of CFU-G (nearly 3fold), CFU-M (3-fold), and CFU-GEMM (3-fold) colonies (Figure 8, $\mathrm{D}-\mathrm{F})$. In addition, $V h l^{R /+}$ splenic cells also gave rise to more CFUerythroid (CFU-E) and CFU-GEMM colonies than did WT cells (Figure 8, D and E). This result supports our earlier findings of a 


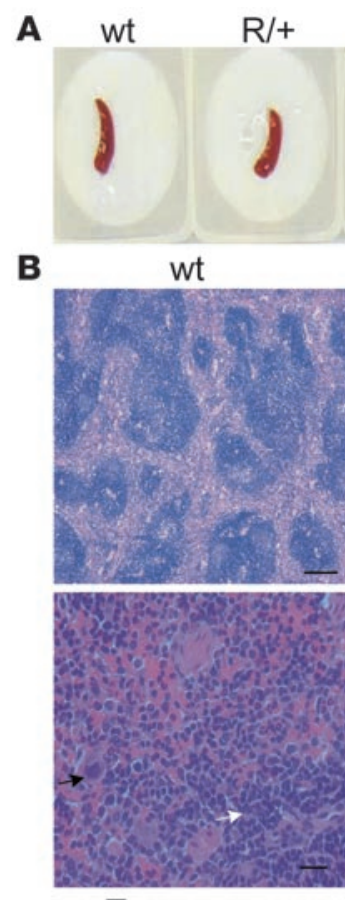

E

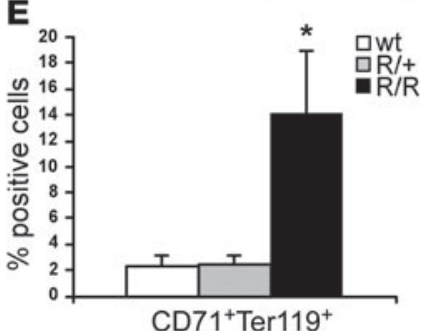

R/+
$\mathrm{R} / \mathrm{R}$ $\mathrm{R} / \mathrm{R}$ R/R
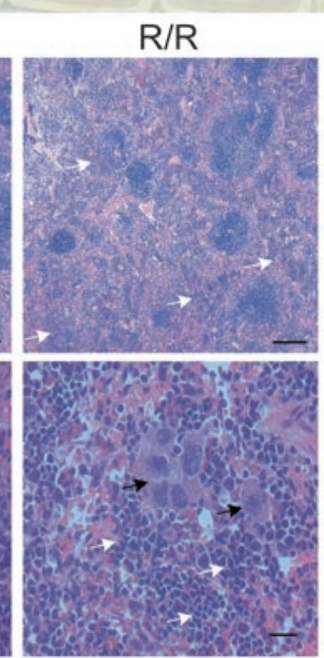

$\mathbf{F}$

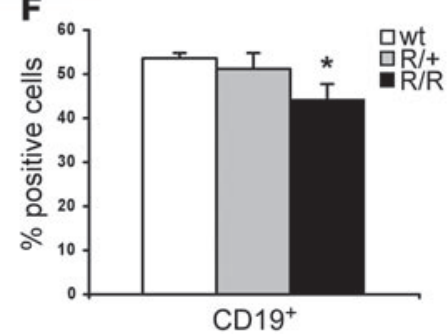

D
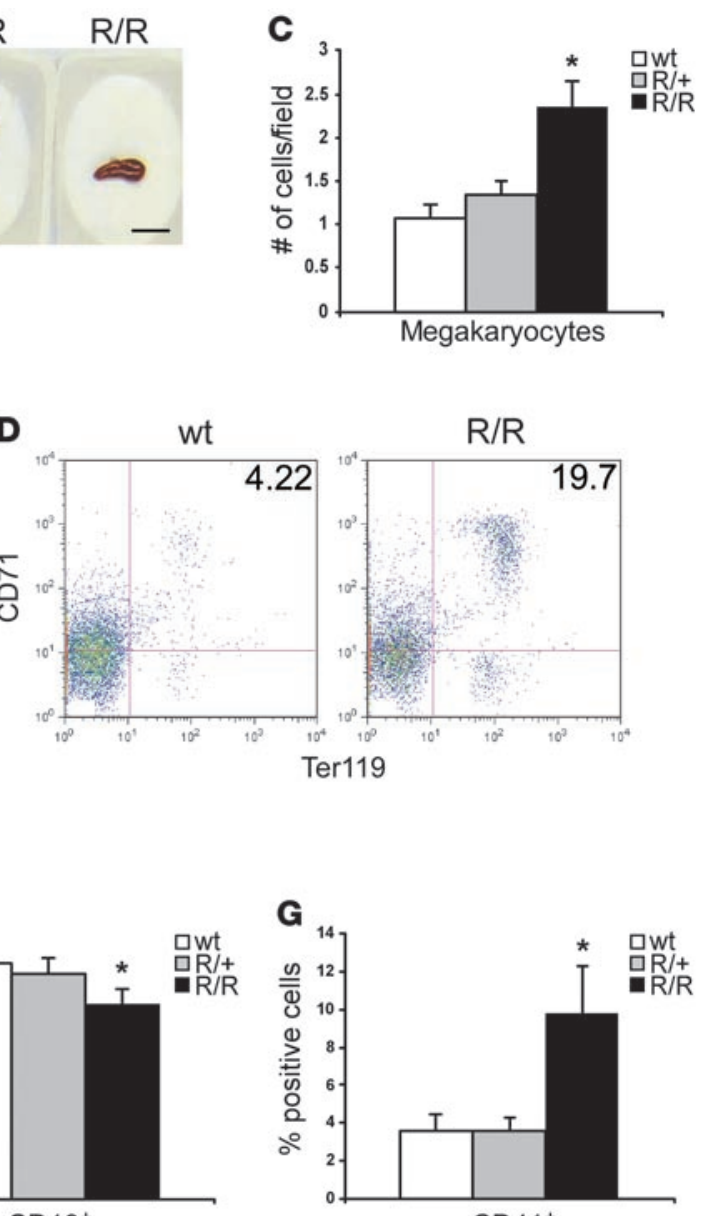

CD41 ${ }^{+}$

\section{Figure 6}

Both erythroid progenitors and megakaryocytes are increased in $V h / R / R$ spleens. (A) Vh/R/R spleens appeared slightly darker in color than WT and $V h /^{R /+}$ littermates. Scale bar: $1 \mathrm{~cm}$. (B) Representative sections of WT and $V h /^{R / R}$ spleens stained with H\&E revealed a disruption of the normal splenic architecture in $V h /^{R / R}$ mice. Erythroid cells are indicated by white arrows, and megakaryocytes by black arrows. Original magnification, $\times 5$ (top); $\times 40$ (bottom). Scale bars: $200 \mu \mathrm{m} ; 20 \mu \mathrm{m}$. (C) Quantification of the increase in megakaryocytes in Vh/R/R mice. ${ }^{*} P<0.006 .(D)$ Representative flow cytometry plots of WT and Vh/R/R spleens stained for CD71 and Ter119. (E and F) The percentage of CD71+Ter119+ erythroid progenitors was increased 6 -fold $(12 \%)$ in $V h / R / R$ spleens $(\mathbf{E})$, corresponding to a $9 \%\left(1.2\right.$-fold) decrease in CD19+ cells $(\mathbf{F})$. ${ }^{*} P<0.05$. $(\mathbf{G})$ The percentage of CD41+ cells was also greater in $V h l^{R / R}$ spleens $\left(2.5\right.$-fold). ${ }^{*} P<0.05$.

larger percentage of erythroid precursors and increased numbers of megakaryocytes in $V h l^{R / R}$ spleens (Figure 6, C and E).

To further assess erythroid differentiation in each organ, we plated bone marrow or splenic cells from each genotype in methylcellulose containing varying concentrations of $\mathrm{EPO}$, without other cytokines. We then scored the number of burst-forming units-erythroid (BFU-E) and CFU-E colonies; BFU-E colonies represent a more immature erythroid precursor and give rise to CFU-E colonies. While the number of CFU-E colonies did not differ with respect to genotype in the bone marrow or spleen (data not shown), the formation of the earlier BFU-E progenitors was greater in $V b l^{R / R}$ mice. There was an increase in the number of BFU-E colonies formed by cells from $V h l^{R / R}$ spleens, as well as $V h l^{R / R}$ bone marrow, at each concentration of EPO tested compared with WT, implying that progenitor cells of mutant mice have an increased sensitivity to EPO (Figure 8, $\mathrm{G}$ and $\mathrm{H}$ ). Collectively, these results suggest that the R200W mutation promotes hematopoietic differentiation, especially of the erythroid lineage, primarily within the spleens of $V h l^{R / R}$ mice.
Increased angiogenesis in $V b l^{R / R}$ mice. In addition to the polycythemic phenotype, we were also interested in identifying other phenotypes in $V h l^{R / R}$ mice that may result from chronic, low-level activation of HIF- $2 \alpha$. In particular, we focused our attention on the skin and liver, based on previous studies demonstrating that HIF- $2 \alpha$ stabilization results in increased vascularity of these tissues (12). Analysis of H\&E-stained skin sections suggested that $V h l^{R / R}$ skin contained more blood vessels than that of WT controls (Supplemental Figure 3A). CD31 staining and quantification of microvessel density confirmed a slight increase in vascularity in $V h l^{R / R}$ skin (Supplemental Figure 3, A and B). This finding is consistent with the enhanced expression of VEGF in $V b l^{R / R}$ mice and may provide an additional explanation for the redness observed in the skin of mutant animals (Supplemental Figure 3A).

We also observed enhanced vascularity in $V h l^{R / R}$ livers, suggesting that VEGF promotes angiogenesis in multiple tissues in mutant mice. Although there were no gross morphological differences nor changes in fat deposition between WT and $V h l^{R / R}$ animals (Supplemental Figure 4A), the number of blood vessels 
A

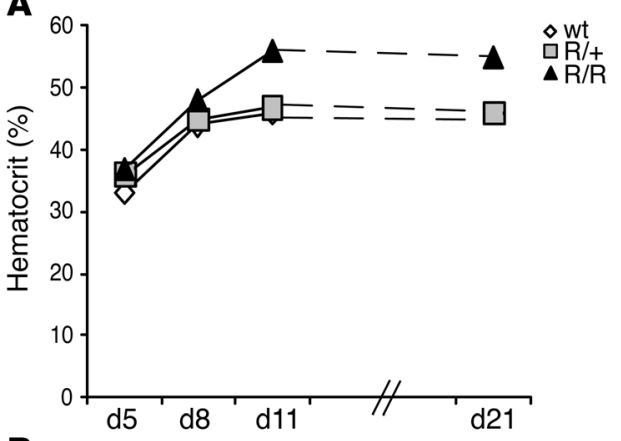

B

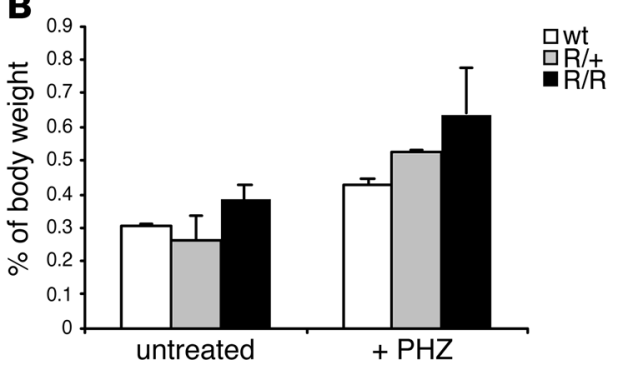

in $V h l^{R / R}$ livers was 1.3-fold greater than that of WT controls (Supplemental Figure 4, A and B). These findings are more moderate than, but in agreement with, the histological changes observed with high-level HIF-2 $\alpha$ activation (12).

Patients with Chuvash polycythemia are also susceptible to developing pulmonary hypertension $(29,31)$, and HIF- $2 \alpha$ has been implicated in hypoxic pulmonary vascular remodeling (35). Therefore, we examined the lungs of $V h l^{R / R}$ mice for signs of pulmonary vascular changes. Staining with $\alpha$-SMA did not reveal discernible differences in the muscularization of vessels. However, the majority of vessels in $V h l^{R / R}$ lungs appeared congested with blood, suggesting the potential existence of pulmonary hypertension in mutant mice (Supplemental Figure 4C).

\section{Discussion}

The $598 \mathrm{C} \rightarrow T$ transition is unique among VHL mutations in that germline homozygosity does not predispose to tumor formation, but instead results in polycythemia $(19,21)$. To gain insight into how this mutation affects $\mathrm{PVHL}$ function, we generated mice expressing the corresponding murine mutation from the endogenous $V b l$ locus. These mice developed polycythemia that became more pronounced with age, although there was no obvious decrease in the life span of these animals. It is worth noting that the fold increase in several parameters, most notably serum EPO levels, was more moderate than the increase seen in patients, likely because of differences between species. Importantly, however, the $V b l^{R / R}$ phenotype qualitatively recapitulated many of the characteristics of Chuvash polycythemia in humans, including increased hematocrits and elevated serum levels of VEGF and EPO, and represents the most accurate mouse model of a VHL-associated disease created to date.

In agreement with previous studies $(19,26)$, the expression of HIF targets, particularly VEGF, EPO, and PAI-1, was enhanced at the mRNA and protein level in $V h l^{R / R}$ ES cells and tissues. Upregulation of these and other genes likely plays a critical role in increased red blood cell formation and the thrombotic tendencies observed in Chuvash polycythemia. Therefore, as suggested by

\section{Figure 7}

Characterization of stress-induced erythropoiesis in $V h /^{R / R}$ mice. (A) Hematocrit of $V h I^{R / R}$ mice, similar to that of WT mice, increased in response to $\mathrm{PHZ}$ (injected on days 1 and 2). However, the erythropoietic response was not downregulated appropriately in $V h R^{R / R}$ mice, and hematocrits continued to rise beyond WT levels to reach $V h l^{R / R}$ pre$\mathrm{PHZ}$ levels. The result of 1 representative experiment is shown; similar results were obtained in separate experiments. (B) Spleen/body weight ratios in untreated mice and mice one month after $\mathrm{PHZ}$ injection; pooled results from multiple experiments are shown. Although the spleens of untreated $V h / R / R$ mice were slightly larger $(0.38 \%$ versus $0.3 \%)$, this difference was enhanced following $\mathrm{PHZ}$, resulting in a greater increase in the size of mutant spleens compared with WT spleens.

Ang et al., a mild dysregulation in the HIF pathway downstream of $\mathrm{Vbl}$ mutation promotes the development of polycythemia (19). However, the potential importance of alterations in HIF-independent functions of PVHL remains to be determined and will be the subject of future investigation using our mouse model.

In contrast to previous studies $(19,26)$, however, HIF- $2 \alpha$ seems to be preferentially activated by this mutation, as normoxic HIF- $2 \alpha$ protein levels were more substantially increased in $V h l^{R / R} \mathrm{ES}$ cells than levels of HIF-1 $\alpha$. Furthermore, many of the genes activated in $V h l^{R / R}$ tissues, including those encoding VEGF, EPO, and PAI-1, have been shown to be HIF- $2 \alpha$-preferential targets in that they are more sensitive to regulation by this $\operatorname{HIF}-\alpha$ isoform $(7,11,12)$. It is possible that HIF-2 $\alpha$ stabilization is more sensitive to perturbations in PVHL function and that upregulation of HIF- $2 \alpha$-specific genes is essential for the development of Chuvash polycythemia. In support of this, mice in which $V b l$ is specifically inactivated in hepatocytes develop polycythemia that is rescued by inactivation of HIF- $2 \alpha$, but not HIF- $1 \alpha$ (9). Similarly, mice expressing constitutively stabilized HIF- $2 \alpha$, but not HIF-1 $\alpha$, develop polycythemia (12). Moreover, mice lacking HIF-2 $\alpha$ expression are anemic, suggesting that this protein is necessary for EPO production and red cell development $(10,36)$. Interestingly, serum EPO levels were also slightly elevated in some $V h l^{R /+}$ mice (Supplemental Figure 2B), although these heterozygotes did not exhibit signs of polycythemia. This suggests that activation of additional HIF- $2 \alpha$ targets, including VEGF, PAI-1, and other as-yet unidentified genes, may be more important. Further analysis will be necessary to confirm the relative contributions of each HIF- $\alpha$ protein to the polycythemic phenotype.

In both ES cells and tissues isolated from $V b l^{R / R}$ mice, the R200W mutation decreased the stability of PVHL. This is in contrast to the human protein, which was expressed at the same level as WT pVHL, at least in B lymphocytes (19), and could represent a difference between the murine and human proteins. It also remains possible that the stability of human R200W pVHL is altered in tissues, such as the kidney, liver, and spleen, that are more physiologically relevant in polycythemia. Although mutant $\mathrm{pVHL}$ levels were reduced, our cumulative results strongly support the notion that the polycythemic phenotype results from the R200W mutation.

Our data demonstrate that the R200W mutation promoted erythroid differentiation and that the spleen was the major source of enhanced erythropoiesis in polycythemic $V h l^{R / R}$ mice. This expansion of splenic erythropoiesis likely occurs in response to elevated VEGF and EPO levels downstream of increased HIF signaling in the spleen and in other tissues such as the kidney and liver. Moreover, the increased dose response of $V h l^{R / R}$ progenitor cells is also a feature of Chuvash polycythemia in humans (19). It is interesting 


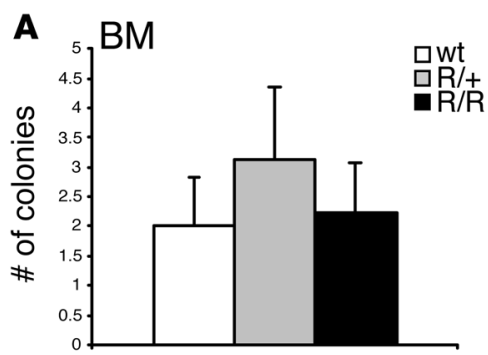

Erythroid

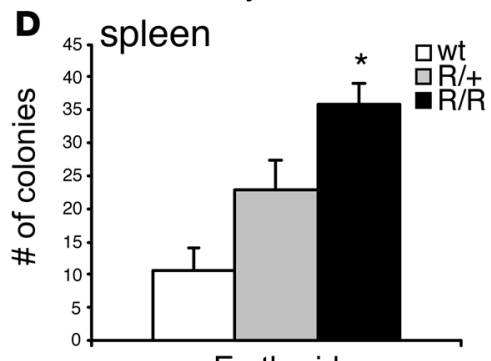

Erythroid

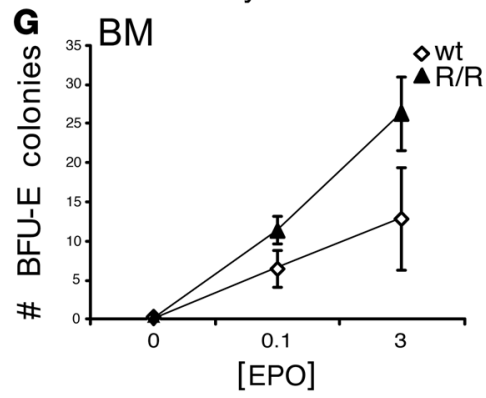

B

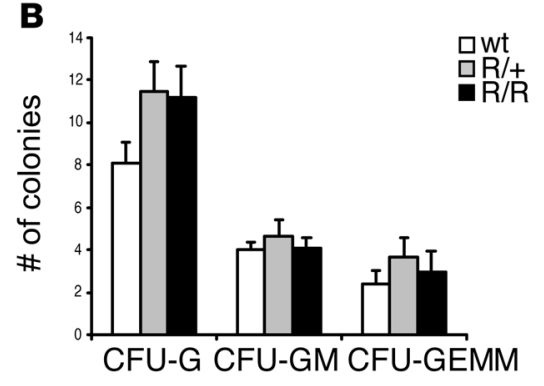

E

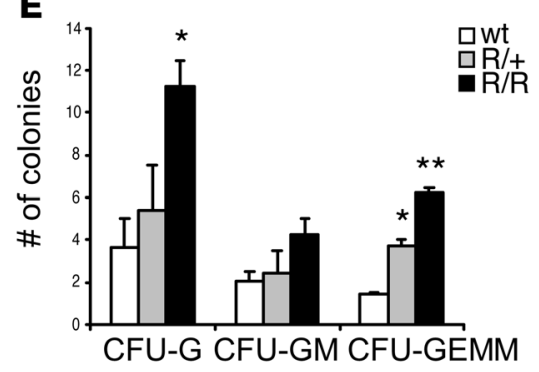

$\mathbf{H}$

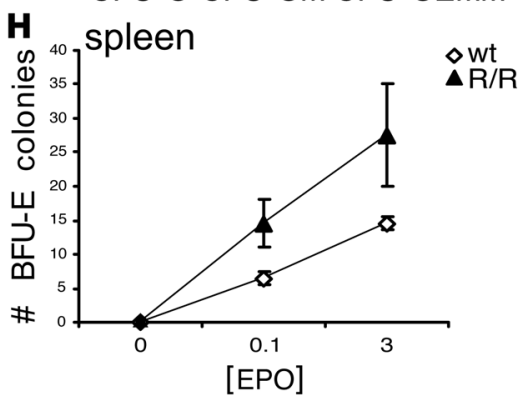

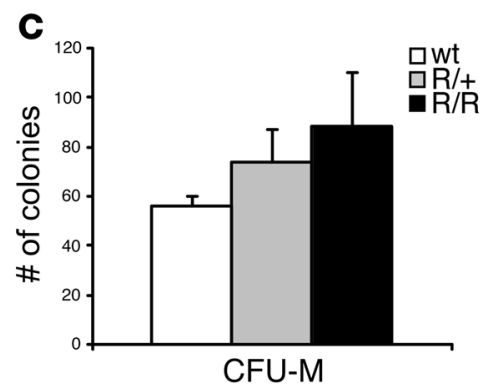

$\mathbf{F}$

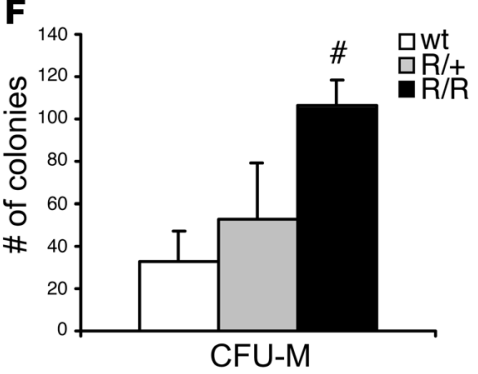

Figure 8

Erythropoiesis is enhanced in $V h l^{R / R}$ spleens in vitro. (A-C) Erythroid differentiation of $V h l^{R / R}$ bone marrow cells was not significantly different from WT (A). Similarly, there was only a small (but not statistically significant) increase in the number of nonerythroid colonies formed by Vh/R/R bone marrow, most notably CFU-G (B) and CFU-M (C). (D-F) In contrast, VhIR/R splenic cells had 3.5-fold higher potential to generate erythroid colonies (D), as well as increased potential to form nonerythroid colonies such as CFU-G and CFU-GEMM (E) and CFU-M (F). ${ }^{*} P<0.02$; ${ }^{* *} P<0.003$; $\# P<0.059$ (trending toward significance). ( $\mathbf{G}$ and $\mathbf{H}$ ) EPO dose-response curves of WT and $V h / R / R$ cells showing the number of BFU-E colonies formed. Both bone marrow $(\mathbf{G})$ and splenic $(\mathbf{H})$ cells were hypersensitive to EPO.

to note that $V h l^{R / R}$ bone marrow was also more sensitive to EPO in vitro, although the extent to which this might contribute to polycythemia remains unclear. Taken together, these results strongly suggest that the spleen is one of the principal tissues affected by this mutation. Splenomegaly has been reported in a small proportion of Chuvash polycythemia patients, but may remain undetected in others $(21,30)$. It will be important to determine whether the spleen is similarly affected in humans and whether this finding has potential therapeutic implications for the treatment of this disease.

The R200W mutation may also have a more general effect on the formation of multiple hematopoietic lineages, particularly megakaryocytes. An increase in megakaryocytes could play a major role in the thrombotic complications that occur in Chuvash polycythemia. This result is especially intriguing because megakaryocytes and erythroid cells originate from the same bipotential megakaryocytic-erythroid progenitor (MEP) (37). Therefore, increased HIF activity at the level of the MEP may stimulate differentiation of both lineages. The finding that immature erythroid progenitors (i.e., BFU-E) seem to be especially sensitive to EPO levels further suggests an effect on the early stages of differentiation. However, the expression of mRNA encoding thrombopoietin (Tpo), a cytokine essential for megakaryocyte differentiation (38), was not increased in $V h l^{R / R}$ livers (data not shown), suggesting that other cytokines such as VEGF and EPO may be more important.

In addition to promoting splenic erythropoiesis, the R200W mutation also enhanced vascularization of several tissues, including the skin and liver. These findings are consistent with previous studies (12) and support the notion that HIF-2 $\alpha$ activation is sufficient to induce angiogenesis, in large part through increased expression of VEGF. Furthermore, this mutation may also result in pulmonary vascular changes, as $V h l^{R / R}$ mice exhibit signs of possible pulmonary hypertension. More detailed histological analysis and physiological measurements are currently in progress to characterize this phenotype and determine whether it recapitulates the cardiopulmonary dysfunction observed in humans with Chuvash polycythemia $(29,31)$.

Our results demonstrate that $V h l^{R / R}$ mice faithfully recapitulate nearly all aspects of Chuvash polycythemia in humans. Additional analysis of this phenotype will provide further insight into how the R200W mutation alters PVHL functions, both HIF-dependent and HIF-independent, to give rise to polycythemia, and will serve as an interesting comparison to mice expressing other $\mathrm{Vhl}$ mutations. 
Furthermore, this model may have implications for our understanding of distinct types of polycythemia and will be useful as a tool to explore potential avenues for the treatment of patients.

\section{Methods}

Generation of $V h l^{R / R}$ mice. To construct the targeting vector, a DNA fragment containing exons 2 and 3 of murine $V h l$ contiguously was digested with BamHI to separate the 2 exons. The exon 2 BamHI fragment was subcloned into the pLNT targeting vector (39) between the HSV-TK gene and floxed $n e o^{R}$ cassette. Clones with the correct orientation were confirmed by restriction digestion and sequencing. Site-directed mutagenesis of $V b l$ exon 3 in pBluescript KS+ (Stratagene) was conducted using the Transformer mutagenesis kit (Clontech) (primer 1, 5'-GGATCCACTAGTTCTGGAGCGCCGCACCGCGGTG-3'; primer 2, 5'-CGAAGGACATACAGTGGCTGAGCCAAGAGCACCTTGAGAGTC-3') and confirmed by bidirectional sequencing. Mutated exon 3 was then directionally cloned as a XhoI/NotI fragment into the pLNT vector containing the exon 2 fragment, adjacent to the floxed $n e o^{R}$ cassette. The completed targeting construct included 3 $\mathrm{kb}$ of homology upstream of exon 2 and $4 \mathrm{~kb}$ of homology downstream of exon 3, and a PvuII/ClaI fragment was used to electroporate J1 ES cells. Doubly gancyclovir- and neomycin-resistant ES clones were selected and screened for proper recombination by Southern blot analysis using HindIII (Roche) digestion and the probe shown in Figure 1B.

$V h l^{R /+}$ ES clones were injected into murine blastocysts and transferred to pseudopregnant females. Germline transmission was confirmed by Southern blot. $V h l^{R /}{ }^{+}$mice were then bred to transgenic E2A-Cre mice (34) and subsequently to $\mathrm{C} 57 \mathrm{BL} / 6$ mice to completely delete the $n e o^{R}$ cassette. Interbreeding of $V h l^{R /+}$ progeny generated $V b l^{R / R}$ mice. WT and $V h l^{R /+}$ littermates were used as controls for all experiments. Mice were genotyped by Southern blot and by PCR using primers that differentiate between the WT and mutant alleles (primer 1, 5'-TGTGCCATCCCTCAATGTCGATGGACAGC-3'; primer 2, 5'-CAGAAAGTGGCTCTGTAGTCTTGACCTTG-3'). In addition, the targeted $V b l$ locus was cloned by PCR and sequenced to confirm the presence of the R200W mutation and ensure that no additional mutations had been introduced.

All procedures involving mice were performed in accordance with the NIH guidelines for use and care of live animals and were approved by the University of Pennsylvania Institutional Animal Care and Use Committee (IACUC).

Cell culture. For in vitro studies, $V h l^{R /+}$ ES cells were grown in increasing concentrations of G418 $(2.5-4 \mathrm{mg} / \mathrm{ml})$ to select for homozygous $V b l^{R / R}$ clones (33). A vector expressing Cre recombinase (pCAAGG-NLS-Cre) was then used to remove the $n e o^{R}$ cassette in vitro. For hypoxic experiments, ES cells were grown under $1.5 \% \mathrm{O}_{2}$ for 6 hours. To determine the half-life of pVHL, ES cells were treated with $200 \mu \mathrm{M}$ cycloheximide (Sigma-Aldrich). For proteasome inhibitor studies, ES cells were treated with one of the following: vehicle DMSO, ALLN $(100 \mu \mathrm{M})$, lactacystin $(10 \mu \mathrm{M})$, or MG-132 ( $20 \mu \mathrm{M}$; Calbiochem). To rescue functional Vbl expression in 786-O human renal carcinoma cells, Lipofectamine Plus (Invitrogen) was used to transfect a vector containing either WT Vhl or VhlR200W murine cDNA. Stable clones were selected using $450 \mu \mathrm{g} / \mathrm{ml}$ hygromycin B (Roche).

$R N A$ isolation, Northern blot analysis, and real-time PCR. Total RNA was prepared from cells or organs using TRIzOL (Invitrogen). RNA from ES cells $(15 \mu \mathrm{g})$ was screened by Northern blot as previously described (7) using the probe shown in Figure 1B or a probe for $\beta$-tubulin as a loading control. For real-time PCR, cDNA was synthesized from $2 \mu \mathrm{g}$ RNA using the SuperScript First-Strand Synthesis System (Invitrogen). Real-time PCR was performed using an ABI Prism 7000 Sequence Detection System with TaqMan Universal PCR Master Mix and TaqMan Gene Expression Assays for HIF target genes (Applied Biosystems). Relative quantification of mRNA expression levels was determined and normalized to $18 \mathrm{~S}$.
Protein analysis. Whole-cell protein extracts were isolated as previously described from ES cells (39) and from organs (40). For supplemental experiments, WT or R200W pVHL was in vitro transcribed and translated using the TNT T7 Quick Coupled kit (Promega) and analyzed by Western blot or exposed to autoradiographic film (Kodak) to detect ${ }^{35} \mathrm{~S}$. The antibodies used for Western blot were as follows: murine HIF-1 $\alpha$ (41), murine HIF-2 $\alpha$ (NB 100-122; Novus Biologicals), murine VHL (M20 and FL-181; both from Santa Cruz; and for tissues 2738; Cell Signaling), ERK (Cell Signaling), eEF2 (Cell Signaling), Akt (Cell Signaling), actin (Sigma-Aldrich), cyclin D1 (DCS-6; NeoMarkers), and p53 (CM5; Novocastra Laboratories). Quantitation of $\mathrm{pVHL}$ levels was performed using NIH Image software.

EPO and VEGF ELISA. Blood collected retro-orbitally was allowed to clot overnight at $4{ }^{\circ} \mathrm{C}$, and serum was isolated by centrifugation for 20 minutes at 2,000 g. Alternatively, supernatants were collected from ES cells grown under normoxia or $1.5 \%$ hypoxia for 24 hours. ELISAs were performed according to the protocols provided by the manufacturer (R\&D Systems).

Phenylhydrazinetreatment. Mice were injected intraperitoneally with $50 \mathrm{mg} / \mathrm{kg}$ phenylhydrazine hydrochloride (Sigma-Aldrich) in PBS on days 1 and 2. Blood was collected retro-orbitally on the day before injection and on every third day after injection, beginning on day 5 ( 3 days after PHZ).

Hematological and histological analyses. Blood was collected by retro-orbital bleed in lithium heparin-treated tubes (BD) for determination of spun hematocrits using microcapillary tubes (Drummond) and an IEC MicroMB centrifuge. Complete blood counts were determined using blood collected in EDTA-coated tubes (Sarstedt) and a Hemavet HV950FS machine (Drew Scientific). For flow cytometry analyses, bone marrow and splenic cells were isolated as previously described (10). Cells were blocked for 15 minutes with CD16/CD32 antibody (1:100 dilution; BD Biosciences - Pharmingen) in $0.1 \%$ BSA in PBS, followed by staining for 30 minutes with primary antibodies: PE-conjugated Ter119, FITC-conjugated CD71, PE-conjugated CD19, or FITC-conjugated CD41 (1:100 dilution; all BD Biosciences - Pharmingen). Analysis was performed on a FACSCalibur machine (BD).

For histology, sections of mouse organs were stained with H\&E. The number of megakaryocytes in the spleen was quantitated from 10 independent high-power fields per slide. Immunohistochemistry was performed using antibodies to CD31 (1:100 dilution; Abcam) or $\alpha$-SMA (1:2,000; Sigma-Aldrich) following antigen retrieval by boiling in sodium citrate solution, pH 6.0 (Vector Laboratories). Microvessel density was expressed as the number of vessels per high-powered field. Staining with oil red O (Sigma-Aldrich) was performed on frozen liver sections.

Colony-forming assays. Bone marrow and splenic cells were isolated as previously described (10). To determine the number of all CFU colonies, $2.5 \times 10^{4}$ bone marrow cells or $2.5 \times 10^{6}$ splenic cells were plated in $2 \mathrm{ml}$ complete Methocult M3434 (StemCell Technologies). EPO dose curves were obtained by plating $1.5 \times 10^{6}$ bone marrow cells or $2.5 \times 10^{6}$ splenic cells in $2 \mathrm{ml}$ Methocult M3234 supplemented with 0.1 or $3 \mathrm{U} / \mathrm{ml}$ recombinant human EPO (both StemCell Technologies). All colonies were scored 6-7 days after plating.

Statistics. Statistical analyses were performed using unpaired Student's $t$ tests, and $P$ values less than 0.05 were considered statistically significant. Error bars in figures represent SEM.

\section{Acknowledgments}

This work was supported by NIH grant 66310 (M.C. Simon), the Abramson Family Cancer Research Institute, and American Cancer Society grant RSG-06189-01-MGO (to W.K. Rathmell). M.C. Simon is an Investigator of the Howard Hughes Medical Institute. M.M. Hickey and N.A. Bezman are supported by Howard Hughes Medical Institute Predoctoral Fellowships in Biological Sciences. The authors would like to thank Aneesa Sataur, Bryan Barnhart, 
Jean Richa, Hongwei Yu, Ed Morrisey, Volker Haase, and Brian Keith for technical assistance, thoughtful discussions, and critical review of the manuscript.

Received for publication May 7, 2007, and accepted in revised form August 29, 2007.

1. Lonergan, K.M., et al. 1998. Regulation of hypoxiainducible mRNAs by the von Hippel-Lindau tumor suppressor protein requires binding to complexes containing elongins B/C and Cul2. Mol. Cell. Biol. 18:732-741.

2. Iwai, K., et al. 1999. Identification of the von Hippel-lindau tumor-suppressor protein as part of an active E3 ubiquitin ligase complex. Proc. Natl. Acad. Sci.U. S. A. 96:12436-12441.

3. Lisztwan, J., Imbert, G., Wirbelauer, C., Gstaiger, M., and Krek, W. 1999. The von Hippel-Lindau tumor suppressor protein is a component of an E3 ubiquitin-protein ligase activity. Genes Dev. 13:1822-1833.

4. Maxwell, P.H., et al. 1999. The tumour suppressor protein VHL targets hypoxia-inducible factors for oxygen-dependent proteolysis. Nature. 399:271-275.

5. Ohh, M., et al. 2000. Ubiquitination of hypoxiainducible factor requires direct binding to the betadomain of the von Hippel-Lindau protein. Nat. Cell Biol. 2:423-427.

6. Semenza, G.L. 2001. Hypoxia-inducible factor 1: oxygen homeostasis and disease pathophysiology. Trends Mol. Med. 7:345-350.

7. Hu, C.J., Wang, L.Y., Chodosh, L.A., Keith, B., and Simon, M.C. 2003. Differential roles of hypoxiainducible factor 1alpha (HIF-1alpha) and HIF2alpha in hypoxic gene regulation. Mol. Cell Biol. 23:9361-9374.

8. Covello, K.L., et al. 2006. HIF-2alpha regulates Oct4: effects of hypoxia on stem cell function, embryonic development, and tumor growth. Genes Dev. 20:557-570.

9. Rankin, E.B., et al. 2007. Hypoxia-inducible factor2 (HIF-2) regulates hepatic erythropoietin in vivo. J. Clin. Invest. 117:1068-1077. doi:10.1172/JCI30117.

10. Gruber, M., etal. 2007. Acute postnatal ablation of Hif2alpha results in anemia. Proc. Natl. Acad. Sci. U. S. A. 104:2301-2306.

11. Raval, R.R., et al. 2005. Contrasting properties of hypoxia-inducible factor 1 (HIF-1) and HIF-2 in von Hippel-Lindau-associated renal cell carcinoma. Mol. Cell. Biol. 25:5675-5686.

12. Kim, W.Y., et al. 2006. Failure to prolyl hydroxylate hypoxia-inducible factor alpha phenocopies VHL inactivation in vivo. EMBO J. 25:4650-4662.

13. Kaelin, W.G., Jr. 2002. Molecular basis of the VHL hereditary cancer syndrome. Nat. Rev. Cancer. 2:673-682.

14. Richards, F.M. 2001. Molecular pathology of von
Address correspondence to: M. Celeste Simon, Howard Hughes Medical Institute, Abramson Family Cancer Research Institute, University of Pennsylvania School of Medicine, BRB II/III, Room 456, 421 Curie Boulevard, Philadelphia, Pennsylvania 19104, USA. Phone: (215) 746-5532; Fax: (215) 746-5511; E-mail: celeste2@mail.med.upenn.edu.
HippelLindau disease and the VHL tumour suppressor gene. Expert Rev. Mol. Med. 2001:1-27.

15. Ohh, M., et al. 1998. The von Hippel-Lindau tumor suppressor protein is required for proper assembly of an extracellular fibronectin matrix. Mol. Cell. 1:959-968.

16. Bishop, T., et al. 2004. Genetic analysis of pathways regulated by the von hippel-lindau tumor suppressor in Caenorhabditis elegans. PLoS Biol. 2:e289.

17. Lee, S., et al. 2005. Neuronal apoptosis linked to EglN3 prolyl hydroxylase and familial pheochromocytoma genes: developmental culling and cancer. Cancer Cell. 8:155-167.

18. Tang, N., Mack, F., Haase, V.H., Simon, M.C., and Johnson, R.S. 2006. pVHL function is essential for endothelial extracellular matrix deposition. $\mathrm{Mol}$. Cell. Biol. 26:2519-2530.

19. Ang, S.O., et al. 2002. Disruption of oxygen homeostasis underlies congenital Chuvash polycythemia. Nat. Genet. 32:614-621.

20. Ang, S.O., et al. 2002. Endemic polycythemia in Russia: mutation in the VHL gene. Blood Cells Mol. Dis. 28:57-62.

21. Gordeuk, V.R., et al. 2004. Congenital disorder of oxygen sensing: association of the homozygous Chuvash polycythemia VHL mutation with thrombosis and vascular abnormalities but not tumors. Blood. 103:3924-3932.

22. Haase, V.H., Glickman, J.N., Socolovsky, M., and Jaenisch, R. 2001. Vascular tumors in livers with targeted inactivation of the von Hippel-Lindau tumor suppressor. Proc. Natl. Acad. Sci. U. S. A. 98:1583-1588.

23. Rankin, E.B., et al. 2005. Inactivation of the arylhydrocarbon receptor nuclear translocator (Arnt) suppresses von Hippel-Lindau disease-associated vascular tumors in mice. Mol. Cell. Biol. 25:3163-3172.

24. Percy, M.J., et al. 2003. Chuvash-type congenital polycythemia in 4 families of Asian and Western European ancestry. Blood. 102:1097-1099.

25. Sergeyeva, A., et al. 1997. Congenital polycythemia in Chuvashia. Blood. 89:2148-2154.

26. Perrotta, S., et al. 2006. Von Hippel-Lindau-dependent polycythemia is endemic on the island of Ischia: identification of a novel cluster. Blood. 107:514-519.

27. Pastore, Y., et al. 2003. Mutations of von HippelLindau tumor-suppressor gene and congenital polycythemia. Am. J. Hum. Genet. 73:412-419.

28. Pastore, Y.D., et al. 2003. Mutations in the VHL gene in sporadic apparently congenital polycythemia. Blood. 101:1591-1595.

29. Bushuev, V.I., et al. 2006. Endothelin-1, vascular endothelial growth factor and systolic pulmonary artery pressure in patients with Chuvash polycythemia. Haematologica. 91:744-749.

30. Gordeuk, V.R., and Prchal, J.T. 2006. Vascular complications in Chuvash polycythemia. Semin. Thromb. Hemost. 32:289-294.

31. Smith, T.G., et al. 2006. Mutation of von HippelLindau tumour suppressor and human cardiopulmonary physiology. PLoS Med. 3:e290.

32. Lewis, M.D., and Roberts, B.J. 2004. Role of the Cterminal alpha-helical domain of the von HippelLindau protein in its E3 ubiquitin ligase activity. Oncogene. 23:2315-2323.

33. Mortensen, R.M., Conner, D.A., Chao, S., Geisterfer-Lowrance, A.A., and Seidman, J.G. 1992. Production of homozygous mutant ES cells with a single targeting construct. Mol. Cell. Biol. 12:2391-2395.

34. Lakso, M., et al. 1996. Efficient in vivo manipulation of mouse genomic sequences at the zygote stage. Proc. Natl. Acad. Sci. U. S. A. 93:5860-5865.

35. Brusselmans, K., et al. 2003. Heterozygous deficiency of hypoxia-inducible factor-2alpha protects mice against pulmonary hypertension and right ventricular dysfunction during prolonged hypoxia. J. Clin. Invest. 111:1519-1527. doi:10.1172/JCI200315496.

36. Scortegagna, M., et al. 2005. HIF-2alpha regulates murine hematopoietic development in an erythropoietin-dependent manner. Blood. 105:3133-3140.

37. Akashi, K., Traver, D., Miyamoto, T., and Weissman, I.L. 2000. A clonogenic common myeloid progenitor that gives rise to all myeloid lineages. Nature. 404:193-197.

38. Kaushansky, K. 1995. Thrombopoietin: the primary regulator of platelet production. Blood. 86:419-431.

39. Covello, K.L., Simon, M.C., and Keith, B. 2005. Targeted replacement of hypoxia-inducible factor-1alpha by a hypoxia-inducible factor-2alpha knock-in allele promotes tumor growth. Cancer Res. 65:2277-2286.

40. Wiesener, M.S., et al. 1998. Induction of endothelial PAS domain protein-1 by hypoxia: characterization and comparison with hypoxia-inducible factor1alpha. Blood. 92:2260-2268.

41. Mansfield, K.D., et al. 2005. Mitochondrial dysfunction resulting from loss of cytochrome c impairs cellular oxygen sensing and hypoxic HIFalpha activation. Cell Metab. 1:393-399. 